\title{
Detection of Sympathetic Activation through Measurement and Adaptive Processing of the Pupil Diameter for Affective Assessment of Computer Users
}

\author{
Ying Gao ${ }^{1}$, Armando Barreto ${ }^{1,2}$ and Malek Adjouadi ${ }^{1,2}$ \\ ${ }^{1}$ Department of Electrical and Computer Engineering, Florida International University, Miami, FL 33174 USA \\ ${ }^{2}$ Department of Biomedical Engineering, Florida International University, Miami, FL 33174 USA \\ *Corresponding Author \\ Armando Barreto \\ 10555 West Flagler Street EC-3956 \\ ECE/BME Departments \\ Florida International University \\ Miami, FL, 33174 \\ Phone: $305-348-3711$ \\ Fax: 305-348-3707 \\ E-mail address: barretoa@fiu.edu
}

Received: 9 April 2009; | Revised: 17 May 2009; | Accepted: 28 May 2009

\begin{abstract}
Future human-computer interactions could be enhanced by enabling the computer to detect the user's emotional states (e.g. stress), and adjust its interaction behavior appropriately. However, providing the computer with the ability to detect important changes in the user's affective state is a challenging problem. We propose that increases in sympathetic activation which typically accompany the onset of stress could be detected noninvasively by monitoring and processing of the pupil diameter (PD) in the computer user. Our previous work indicated that, under constant illumination conditions, there are measurable increases of the pupil diameter in response to stress stimuli, which we have termed the Pupillary Affective Response (PAR). On the other hand, it is known that the Pupillary Light Reflex (PLR) plays a significant role in modifying the pupil diameter and, therefore, the PAR must be separated from the PLR to be useful towards affective assessment of a computer user in environments with variable illumination. To that end, this study adds the measurement of illumination (IL) in the neighborhood of the subject's eyes and applies it to the processing of the PD signal through an Adaptive Interference Canceller (AIC) using the $\mathrm{H}^{\infty}$ time-varying (HITV) adaptive algorithm, to remove the influence of the PLR from the pupil diameter variations originally measured. The output of the AIC, the Modified Pupil Diameter (MPD) signal, is subjected to additional processing, to result in a detection feature that is compared against a threshold to obtain an indication of subject "stress". The performance of this stress detector, along with 9 others derived from Galvanic Skin Response (GSR) and Blood Volume Pulse (BVP) signals is studied through their Receiver Operating Characteristic (ROC) curves.
\end{abstract}

Keywords: Pupil Diameter (PD); Sympathetic Activation; Adaptive Interference Canceller (AIC) 


\section{Introduction}

Research is currently underway to enhance the interaction between humans and computers by providing computers with the ability to recognize their users' affective states (e.g., stress), so that computers may adjust their interaction behavior accordingly [1][2]. In part, this has been attempted by monitoring and analyzing physiological signals which are inherently controlled by the Autonomic Nervous System (ANS), such as the Galvanic Skin Response (GSR), the Blood Volume Pulse (BVP), and, recently, the Pupil Diameter (PD). This approach is based on the well-established link between the psyche of a human being and his/her physiological processes through the Sympathetic and Parasympathetic divisions of the ANS [3]. Such a method seems particularly suitable when the goal of affective sensing is focused, for example, on the differentiation of states of relaxation (known to be associated with parasympathetic preponderance) from states of stress (identified with sympathetic activation).

Traditional affective assessment methods based on physiological variables utilize signals such as the GSR and the BVP. Some recent approaches have been focused on the variations of pupil diameter associated with sympathetic activation, including our previous work, under constant illumination levels [4].The pupil is the aperture located in the center of the iris, which controls the amount of light that enters the eye. The diameter of this circular aperture is under the control of the ANS through two sets of muscles. The sympathetic ANS branch, mediated by the posterior hypothalamic nuclei, produces enlargement of the pupil by direct stimulation of the dilator muscles, which causes them to contract [5].

On the other hand, pupil contraction is caused by excitation of the circular pupillary constriction muscles innervated by the parasympathetic fibers. The motor nucleus for these muscles is the Edinger-Westphal nucleus located in the midbrain. Sympathetic activation brings about pupillary dilation via two mechanisms: (i) an active component arising from activation of radial pupillary dilator muscles along sympathetic fibers and (ii) a passive component involving inhibition of the Edinger-Westphal nucleus [6].

It is well known that one of the most common and powerful causes for pupil contraction is the Pupillary Light Reflex (PLR), which, may be viewed as a nonlinear, local closed-loop feedback system for regulating the retinal light flux, so that the pupil area is decreased when larger light intensity (luminance) falls on the retina [6]. However, recent studies reveal that the pupil diameter is also modulated by sympathetic activation elicited by affective responses. The impact of affective stimulation over pupil diameter has been verified experimentally by Partala and Surakka, who found that using auditory emotional stimulation, the pupil size variation can be seen as an indication of affective processing [7]. More recently, Steinhauer et al. confirmed that pupil size is associated with the level of difficulty of a task performed by experimental subjects. Using alternate pharmacological blockades of sympathetic and parasympathetic effects on the pupil, they were able to characterize and differentiate the contributions of the two ANS divisions to the control of pupil diameter [5].

Therefore, in our research, we attempt to remove the contribution of the PLR in the measured pupil diameter signal, leaving only components derived from the affective response of the subject, which we have termed the Pupillary Affective Response (PAR). We approach this problem by processing the measured Pupil Diameter signal with an Adaptive Interference Canceller (AIC), and performing additional postprocessing on the resulting Modified Pupil Diameter (MPD), in order to derive a sequence of values (Processed MPD, or PMPD) that can be compared against a threshold to perform as a stress detector.

The adaptive interference canceller is a system known to be able to remove an unwanted interference component $z(k)$ that pollutes a signal of interest $s(k)$ using an independent measurement of the interference $n(k)$ [8]. Therefore, in order to apply an AIC system to the problem at hand, it is necessary to obtain a simultaneous measurement of the illumination reaching the area around the 
subject's eye, to be used as the "independent measurement of the interference". Additionally, for the purpose of contrasting the affective assessment results derived from the PD signal and affective assessments driven by more traditional signals, we measured and recorded photoplethysmographic BVP and GSR signals simultaneously to the PD recordings, in our experiment, described in the following sections.

\section{Materials and methods}

\subsection{Stress elicitation.}

In order to observe the changes in the PD, BVP and GSR signals and their correlation to sympathetic activation caused by stress, we needed to elicit stress in the experimental subjects during controlled intervals. In this study, the "Stroop Color-Word Interference Test" [9], implemented as a Flash program [10], was used to elicit mild mental stress in the subjects at known times. The efficiency of this stress elicitation method has been previously established by Renaud and Blondin [11].

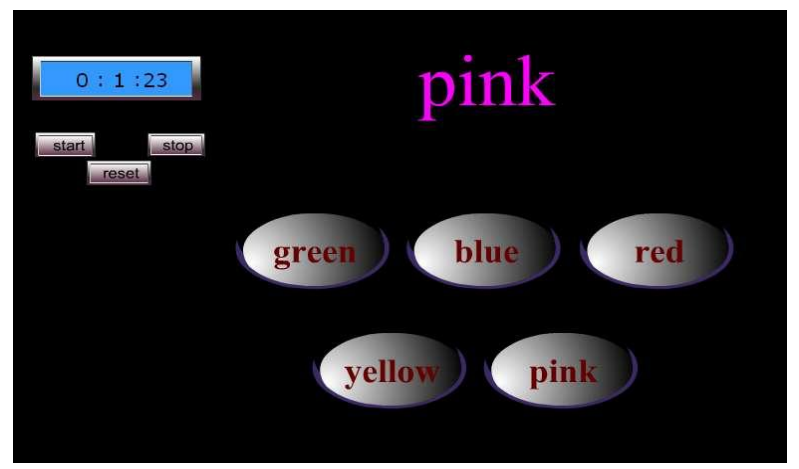

Figure 1. Sample of the Stroop Test interface used for stimulation. The test word appears at the top. The subject was asked to click one of the onscreen buttons to indicate the font color of the word displayed.

In the test, a word presented to the subject was shown in a color that may ("Congruent word presentation") or not ("Incongruent word presentation") match the meaning of it. The subjects were instructed automatically by the program to click one of the five screen buttons to indicate the font color of the word presented. A typical (congruent) example of this test interface is shown in Figure 1.

The complete experiment protocol (Figure 2) comprised three consecutive sections.

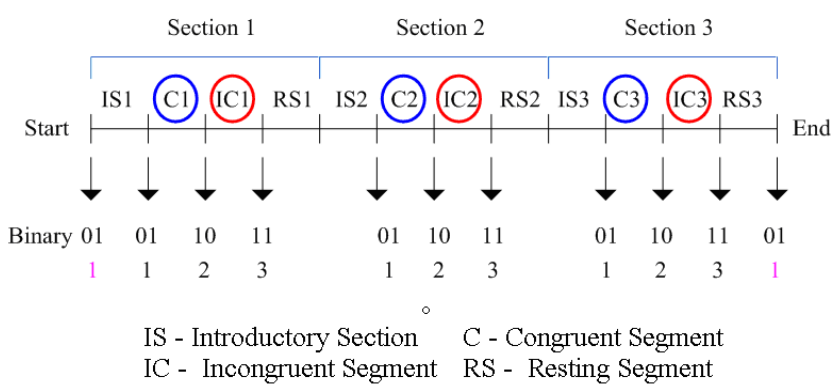

Figure 2. Stimuli schedule of the experimental protocol used. There were 3 consecutive sections with a Congruent Stroop and an Incongruent Stroop segment in each (shown circled).

In each section, there were four segments, including:

- 'IS' - the Introductory Segment to let the subject get used to the task environment, in order to establish an appropriate initial level for his/her psychological state, according to the law of initial values (LIV) [12];

- $\quad$ 'C' - the Congruent segment, comprising 45 Stroop congruent word presentations (font color matches the meaning of the word), which are not expected to elicit significant stress in the subject;

- ' 'IC' - the Incongruent segment, in which the font color and the meaning of the 30 words presented are different. This is expected to induce stress in the subject;

- 'RS' - a Resting Segment to let the subject relax for some time.

At the same time, numerical codes provided by the stimulus generator every time one of the $\mathrm{C}$, IC or RS segments started were used to insert the corresponding values (1, 2 or 3$)$ in the event channel of the PD data file, and the corresponding time marks in the IL, GSR and BVP measurement data, which were recorded simultaneously. The synchronization codes were provided by the Flash stimulus program to the external instrumentation as short bursts of sinusoidal tones appearing in the left and/or right channels of the audio output of the stimulus computer. Thus, simultaneous bursts in both, left and right channels indicated code " 3 " 
("11" in binary). A burst in only the left audio channel (binary "01") was used to indicate "code 1 ". A burst in only the right audio channel (binary "10") was used to indicate "code 2". The simultaneous absence of bursts in the left and right channels (binary " 00 ") was the default condition and, therefore, did not indicate any specific synchronization event. The first and fifth occurrences of "code 1" signaled the beginning and end of the part of the protocol that involved Stroop stimuli (as such they are shown in a different color, in Figure 2).

\subsection{Biosignal recording}

In this study, a desk-mounted eye tracking system (TOBII T60), was used to record the pupil diameter signals from both eyes of the subjects at $60 \mathrm{~Hz}$. The average $((\mathrm{L}+\mathrm{R}) / 2)$ was recorded as the "Measured PD" signal, which corresponds to the "primary input", $d(k)$, for the AIC.

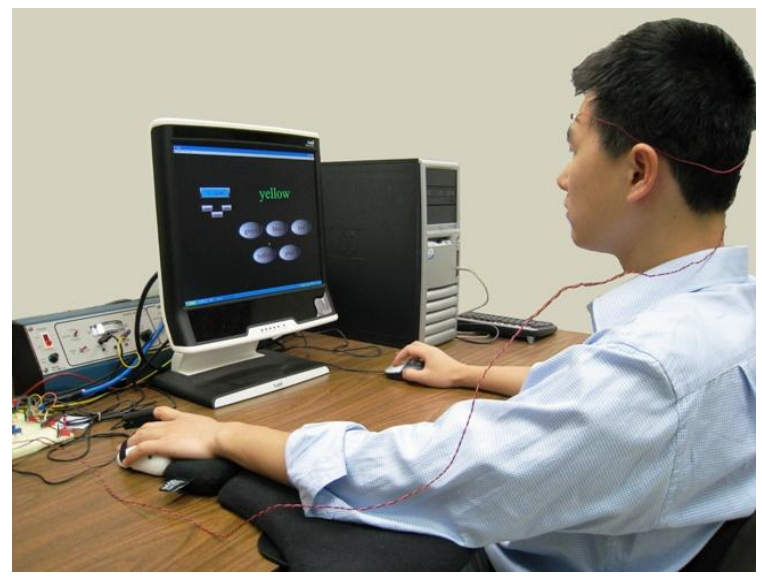

Figure 3. Typical subject placement, in front of the TOBII T60 system, with GSR and BVP sensors in the left hand and illumination sensor on the forehead.

Simultaneously, the Illumination Level (IL), Galvanic Skin Response (GSR) and Blood Volume Pulse (BVP) signals were measured and sampled at the frequency of $360 \mathrm{~Hz}$ with a Data Acquisition (DAQ) System (PCI-DAS6023 board from Measurement Computing Co.). Figure 3 shows the typical placement of an experimental subject in front of the TOBII T60 screen, the GSR and BVP sensors placed in the left hand of the subject, and wires carrying the signal from the illumination sensor in his forehead.

A BS500B0F photo-diode (Sharp), was placed on the forehead of the subject to measure the illumination around the subject's eyes. This sensor was connected to an amplification circuit [13] (shown in Figure 4) to provide an analog output voltage that is proportional to the illumination intensity level $(\sim 0.0043$ volt/lux, where the conversion factor is given according to the units for voltage and illuminance of the International System of Units). The output of the circuit was digitized $(360 \mathrm{~Hz})$ to obtain the "Measured IL" signal, $r(k)$, used as reference sequence for the AIC system.

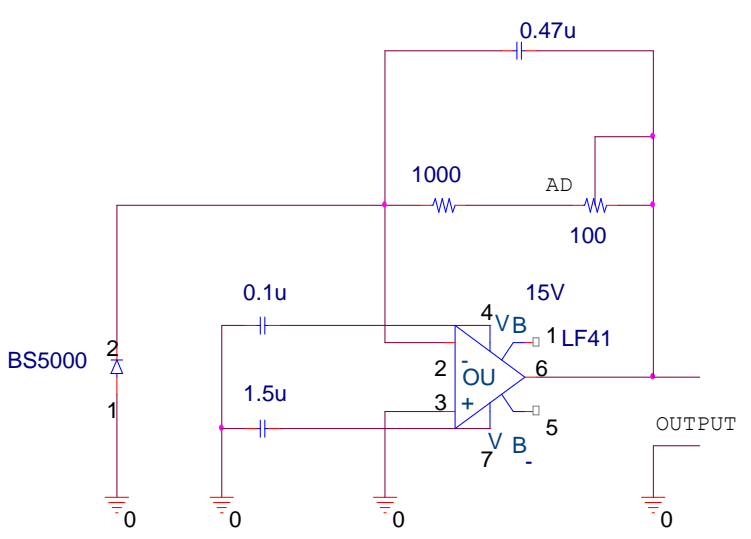

Figure 4. Electronic circuit used for the preprocessing of the signal from the illumination sensor used in the experiments (from [13]).

A GSR2 module (from Thought Technology LTD), combined with a "frequency to voltage converter" integrated circuit (LM2917N) were used to measure the GSR by passing a small current through a pair of electrodes placed on the surface of the skin, measuring the conductivity level, which was converted, proportionally, to voltages.

An infrared finger photoplethysmograph (PPG) (UFI Model 1020), which relies on the light absorption characteristics of blood, was utilized for the measurement of the Blood Volume Pulse (BVP) signal.

In our experiments, 14 adult participants were asked to remain seated in front of the TOBII screen, interacting with the "Stroop Test" program for about 30 minutes, while wearing a head band 
with the photo-diode, the GSR sensor on the middle and ring fingers and the photoplethysmograph on the index finger. During that time, all the normal lights in the room were kept $\mathrm{ON}$, but an additional level of illumination provided by a desk lamp placed above the eye level of the subject was switched ON intermittently. This was achieved by sliding a dimmer abruptly from its minimum position to its maximum position, and vice versa, to repeatedly introduce intervals of higher illumination in the experiment, which would trigger the pupillary light reflex.

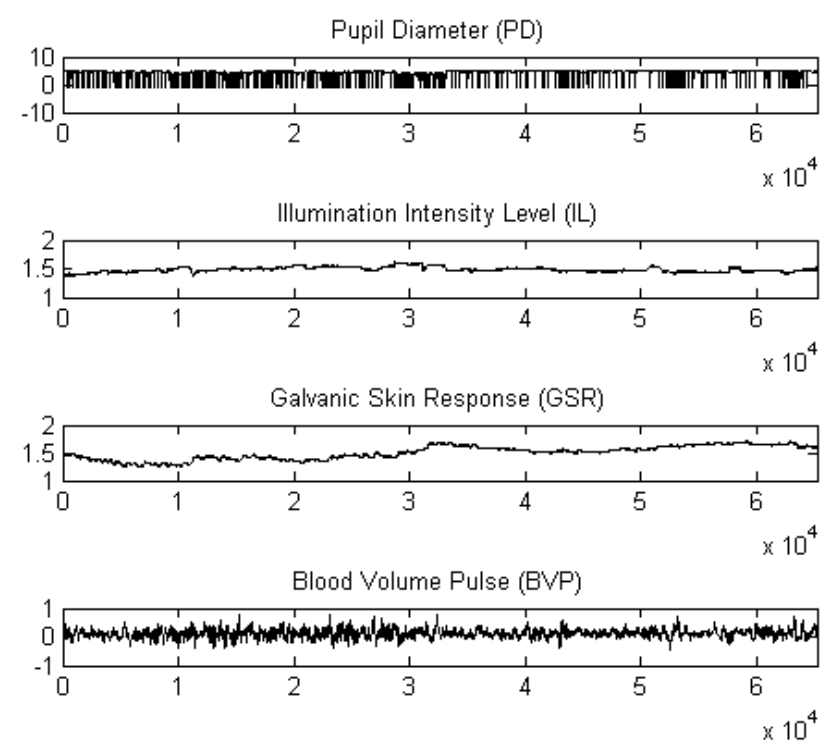

Figure 5. PD, IL, GSR and BVP signals in a complete experimental session. This figure shows that, other than some subtle local GSR variations, the differentiation between congruent and incongruent Stroop segments is not immediately evident from the raw signals.

Figure 5 shows an example of the four signals recorded from a subject through the complete experimental session, once all of the signals have been synchronized at a sampling rate of 60 samples per second, after down-sampling the GSR, BVP and IL signals, to share the same sampling frequency of the PD signal. (The GSR and BVP signals were originally recorded at $360 \mathrm{~Hz}$ to make them suitable for the application of other signal processing algorithms that may require a high sampling rate. However, for this specific research, it was necessary to have the same sampling rate as the PD signal, which is 60 Hz.)

\subsection{Pre-processing of the PD signal}

Prior to the application of the adaptive interference canceller, the interruptions in the PD data, due to blinking (identified as a value of " 4 " in the validity code provided by the TOBII system), were compensated by linear interpolation. The resulting signal was processed by a low pass, $512^{\text {th }}$ order FIR filter with a cutoff frequency of $0.13 \mathrm{~Hz}$ to complete the elimination of the blink responses.

\subsection{Adaptive Interference Cancelling}

The block diagram of the AIC is shown in Figure 6. The PD signal obtained after blinkremoval was applied to the AIC system as the primary input signal $d(k)$, and the reference input $r(k)$ was the simultaneously measured illumination intensity level signal, which was down-sampled from $360 \mathrm{~Hz}$ to $60 \mathrm{~Hz}$ to share the same sampling rate with the PD signal.

For the purpose of interference cancelling, the signal of interest, polluted with an uncorrelated noise signal, is transmitted over the top channel as the primary input to the AIC. The signal applied to the bottom channel, which is uncorrelated with the signal of interest, but correlated with the interference, contributes the reference input. The output of the AIC is expected to provide a signal where the correlated interference has been removed.

The key equations describing the operation of the AIC are:

Primary Input: $d(k)=s(k)+z(k)$

Reference Input: $r(k)=n(k)+u(k)$

Output:

$e(k)=d(k)-y(k)=d(k)-\hat{z}(k)=\hat{s}(k)$

where,

- $s(k)$ : signal of interest (affect-driven PD change), uncorrelated to the reference $(\operatorname{cov}(s(k), r(k))=0)$;

- $z(k)$ : interference in the primary sensor (PD changes driven by PLR);

- $n(k)$ : actual source of the interference (IL 
changes), correlated to $z(k)$, i.e. $(\operatorname{cov}(z(k), r(k)) \neq 0)$;

- $\quad u(k)$ : measurement noise (assumed negligible).

In the AIC system, the core element is an adaptive transversal filter (ATF), where the reference signal $r(k)$ is processed to produce an output $y(k)=\hat{z}(k)$ that is an approximation of $z(k)$. The state space model of the ATF is given by [14]:

$$
\begin{aligned}
& w(k+1)=w(k)+\Delta w(k) \\
& d(k)=r(k)^{T} w(k)+v(k) \\
& z(k)=r(k)^{T} w(k)+v(k) \\
& v(k)=s(k)+v(k)
\end{aligned}
$$

In these equations,

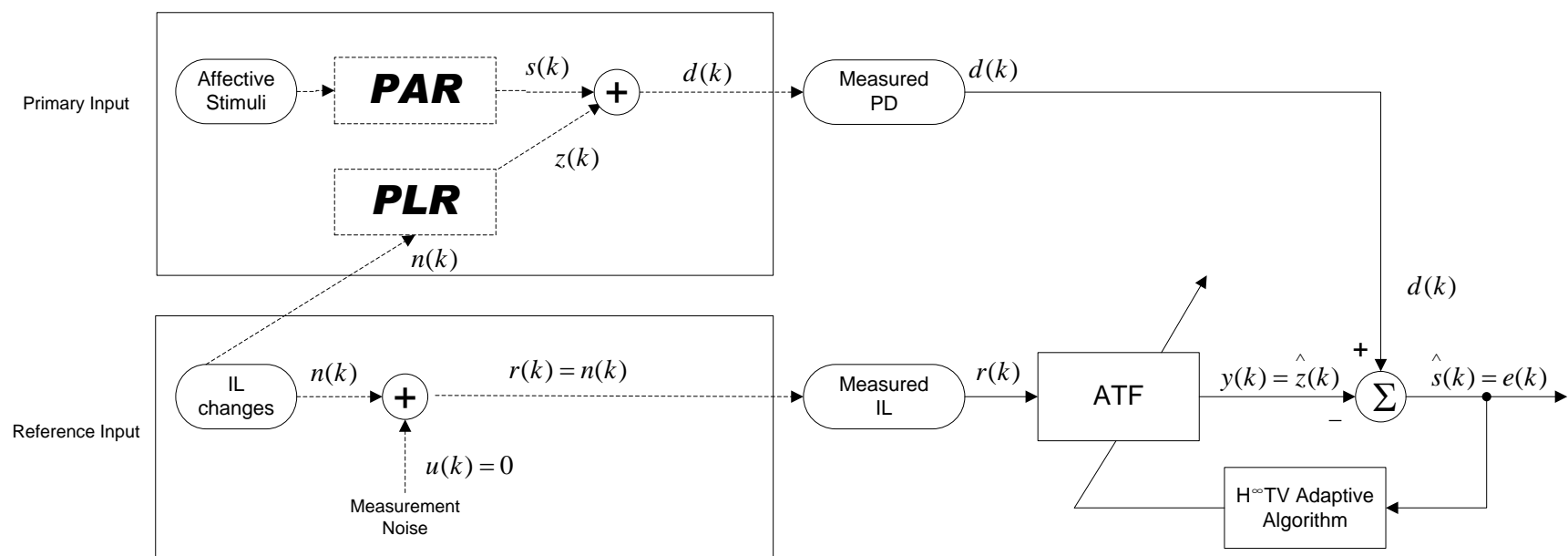

Figure 6. Adaptive Interference Canceller (AIC) Block Diagram. The rectangular boxes on the left illustrate the generation of the measured signals ("Measured PD" and "Measured IL") processed by the AIC , as hypothesized in this study.

In this study, we treated the recorded pupil diameter signal as the primary input of the AIC, which is composed by the signal of interest $s(k)$ (PD- driven by PAR) and the interference $z(k)$ (PD- driven by PLR). In principle, the reference input comprises both, the actual source of the interference $n(k)$ (actual illumination changes) and the measurement noise $u(k)$. However, with the assumption that the measurement noise is negligible in our experimental setup, an independent measurement of illumination in the
- $w(k)=$ system state vector; which is the ATF coefficient vector of size $m \times 1$ ( $\mathrm{m}$ is the order of the ATF);

- $d(k)=$ measurement sequence, which is the observed pupil diameter (PD) signal.

- $z(k)=$ sequence to be estimated

- $\Delta w(k)=$ process noise vector, which represents the time variation of the ATF weights $w(k)$.

- $v(k)=$ measurement noise vector, which includes $s(k)$ (PD- driven by affective changes) and model uncertainties $v(k)$.

- $r(k)=\left[r_{k}, r_{(k-1)}, \ldots \ldots, r_{(k-m+1)}\right]^{T}$, which is the interference vector of size $m \times 1$ 
desired signal $s(k)$, containing only the PD variations due to affective changes (PAR).

The adaptive algorithm we selected for the Adaptive Interference Canceller system, in this study, was the $\mathrm{H}^{\infty}$ time-varying (HITV) adaptive algorithm, which attempts to remove the interference from the recorded signal by adaptively adjusting the impulse response of the ATF [15]. The robustness of this HITV algorithm is derived from its minimization of the maximum energy gain from the disturbances to the estimation errors with the following solutions [14]:

$$
\begin{aligned}
& \tilde{P}^{-1}(k)=\mathrm{P}^{-1}(k)-\varepsilon_{g}^{-2} \mathrm{r}(k) \mathrm{r}^{T}(k) \\
& g(k)=\frac{\tilde{\mathrm{P}}(k) \mathrm{r}(k)}{1+\mathrm{r}^{T}(k) \tilde{\mathrm{P}}(k) \mathrm{r}(\mathrm{k})} \\
& \hat{w}(k+1)=\hat{w}(k)+g(k)\left(y(k)-\mathrm{r}^{T}(k) \hat{w}(k)\right) \\
& P(k+1)=\left[P^{-1}(k)+\left(1-\varepsilon_{g}^{-2}\right) r(k) r^{T}(k)\right]^{-1}+\gamma_{0} \\
& P(0)=\eta I=\Pi_{0}, \Upsilon_{0}=\rho I
\end{aligned}
$$

In these equations, $g(k)$ is the gain factor; $\varepsilon_{g}, \eta$ and $\rho$ are positive constants. Note that $\rho$ reflects a priori knowledge of how rapidly the state vector $w(k)$ varies with time, and $\eta$ reflects the a priori knowledge of how reliable the initial estimate available for the state vector $w(0)$ is.

\subsection{Detection signal definition and normalization}

In this study, our specific aim is to evaluate the affective assessment performance of a singlefeature stress detector, derived from PD, in comparison to 9 other single-feature detectors derived from GSR and BVP. These detectors are assessed in terms of the individual discriminating power to differentiate between the Congruent Stroop segments (associated with "relaxed" affective states in the user) and the Incongruent Stroop segments (which are assumed to have caused "stressed" states in the subject). Table 1 shows the features obtained from each of the signals, resulting in a single feature value for each "C" or "IC" segment in the test. The postprocessing operated on the MPD signal to obtain the PMPDmean feature is detailed in the next section. The processes to obtain the remaining 9 features are reflected in their names (further Am. J. Biomed. Sci. 2009, 1(4), 283-294; doi: 10.5099/aj090400283

\begin{tabular}{|c|c|c|}
\hline Biosignal & Features & Definition \\
\hline $\begin{array}{l}\text { PD } \\
(1 \\
\text { feature })\end{array}$ & PMPDmean & $\begin{array}{l}\text { Average value of the } \\
\text { non-negative MPD } \\
\text { signal after median } \\
\text { processing }\end{array}$ \\
\hline \multirow{5}{*}{$\begin{array}{l}\text { GSR } \\
(5 \\
\text { features })\end{array}$} & GSRmean. & $\begin{array}{l}\text { Average value of the } \\
\text { GSR samples }\end{array}$ \\
\hline & GSRnum & $\begin{array}{l}\text { Number of the GSR } \\
\text { responses }\end{array}$ \\
\hline & GSRmeanAmp & $\begin{array}{l}\text { Mean value of the } \\
\text { amplitude of each } \\
\text { GSR response }\end{array}$ \\
\hline & GSRrisingTime & $\begin{array}{l}\text { Rising time of each } \\
\text { GSR response }\end{array}$ \\
\hline & GSReng & $\begin{array}{l}\text { Energy of GSR } \\
\text { (total under the } \\
\text { rising time curve) }\end{array}$ \\
\hline \multirow{4}{*}{$\begin{array}{l}\text { BVP } \\
(4 \\
\text { features })\end{array}$} & BVPmeanAmp & $\begin{array}{l}\text { Mean value of the } \\
\text { amplitude of each } \\
\text { BVP beat }\end{array}$ \\
\hline & BVPl2h & $\begin{array}{l}\text { Low frequency to } \\
\text { high frequency rate } \\
\text { for Power spectral } \\
\text { density of interbeat } \\
\text { interval(IBI) (the } \\
\text { time in milliseconds } \\
\text { between two normal, } \\
\text { consecutive peaks in } \\
\text { the BVP) sequence }\end{array}$ \\
\hline & BVPIBImean & $\begin{array}{l}\text { Mean value of the } \\
\text { IBI }\end{array}$ \\
\hline & BVPstd & $\begin{array}{l}\text { Standard deviation } \\
\text { of IBI sequence }\end{array}$ \\
\hline
\end{tabular}

details for the calculation of these 9 features can be found in [16]. They are not included in this paper as the emphasis is on the PD signal).

Table 1 Features extracted from the bio-signals

Before attempting to use these features, individually, to identify "stressed" (incongruent) and "relaxed" (congruent) segments in the experiment, each feature was normalized, according to the following steps.

1) Reference the features to their baseline level.

$$
Y_{s}=\frac{X_{s}}{X_{r}}
$$


Here $X_{s}$ is the raw feature value during " $C$ " and "IC" segments, and $X_{r}$ is the corresponding feature value during the relaxation period, prior to the first congruent segment. (This was done only for GSR and BVP features)

2) Minimize the impact of individual subject responses dividing each feature by the sum of all six segment values (3 "C" and 3 "IC")

$$
Y_{s}{ }^{\prime}=\frac{Y_{s i}}{\sum_{i=1}^{6} Y_{s i}}
$$

3) Normalize all data to the range of $[0,1]$ with max-min normalization.

$$
Y_{\text {norm }}=\frac{Y_{s}{ }^{\prime}-Y_{s}{ }^{\prime} \text { min }}{Y_{s}{ }^{\prime}{ }_{\text {max }}-Y_{s}{ }^{\prime} \text { min }}
$$

\subsection{Receiver Operating Characteristic (ROC)}

Curves

In signal detection theory, a Receiver Operating Characteristic (ROC) curve is a graphical representation that illustrates the tradeoff made in a classifier between two operating characteristics, true positive rate (TPR) and false positive rate (FPR), as its discrimination threshold is varied. The TPR refers to the fraction of all positive cases correctly classified; correspondingly, the FPR reflects the false alarm level, i.e., the fraction of negative cases incorrectly classified as positive. In the ROC plot, the origin of the coordinate axes represents the case when the classifier produces no false alarms but detects no positive cases at the lowest sensitivity level (i.e., setting the threshold at the highest possible value of the detection signal). In contrast, the top righthand corner represents the situation when the classifier reports all positive cases correctly (true positive rate $=1$ ), but all the negative cases incorrectly (because it raises a false alarm on each negative case, i.e., false positive rate $=1$ ), when the threshold is set at the lowest possible value of the detection signal.

Generally, the ROC curves "start" at the coordinate origin, $(0,0)$ and "end" at the upperright corner $(1,1)$. The trajectory between these points followed by a given ROC, and consequently, the "Area under the ROC" (AUROC) are indicators of the discriminating power of the detection signal being thresholded.

A classification system based on a highly discriminating detection signal (in our case, a feature) will have one or several threshold levels that map close to the upper-left corner of the ROC, at $(0,1)$. This location represents a high sensitivity (large true positive rate) and also a high specificity (low false positive rate). If that is the case, the AUROC will come close to encompassing the full 1.0-by-1.0 square. That is, the AUROC will approach the ideal value of 1 . On the other hand, an ROC curve that remains close to the bottomright corner implies a lack of discriminating power in the detection signal (feature), and will result in a smaller value of the corresponding AUROC.
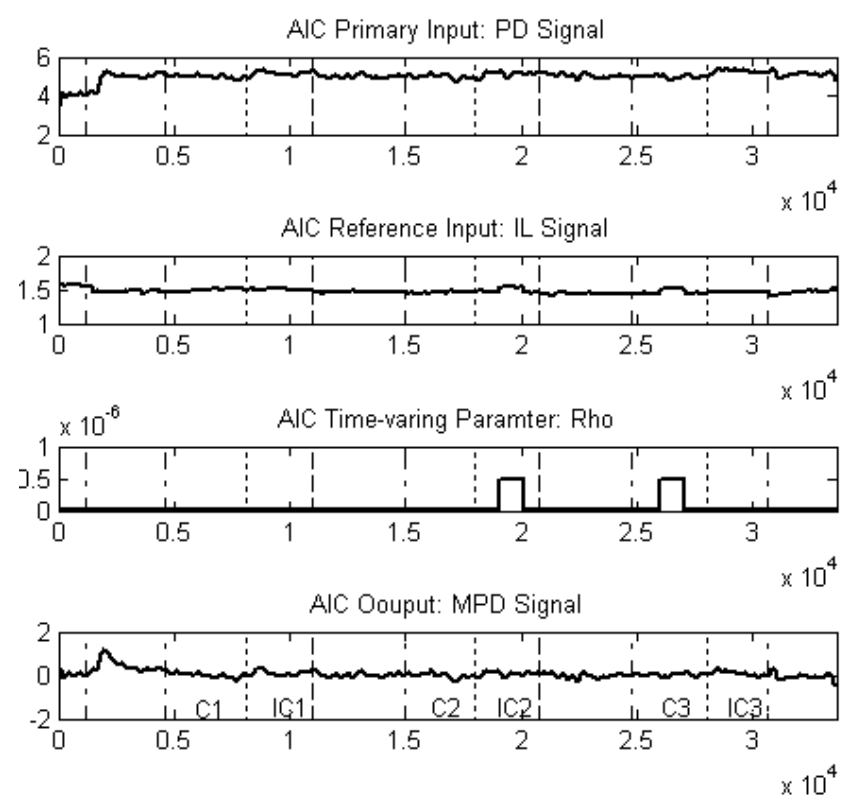

Figure 7. Example of the results from AIC processing of PD with the HITV Algorithm. The top 2 traces represent the signals fed to the AIC. The bottom panel is the output of the AIC (MPD signal).

\section{Results}

\subsection{Results from AIC Processing of PD}

Figure 7 shows an example of the PD signal (after blink removal) and the IL signal. It also shows the increases in the value of the adaptation parameter $(\rho)$ determined by the detection of illumination increases seen in IL. Finally the bottom panel shows the AIC output, i.e., the Modified Pupil Diameter (MPD) signal. 
As mentioned by Beatty and LuceroWagoner, the affective state of "stress" is expected to cause a dilation of the pupil [17]. Therefore, the negative portions of the MPD signal were zeroed out to isolate significant MPD increases, which indicate the emergence of stress in the subject (shown in the middle panel of Figure 8). Then, a sliding window with a width of 900 samples is further applied throughout the non-negative MPD signal to calculate the median value within each window. The outcome of this process can be seen in the bottom panel of Figure 8. The resulting signal is termed the Processed MPD, or "PMPD" signal.
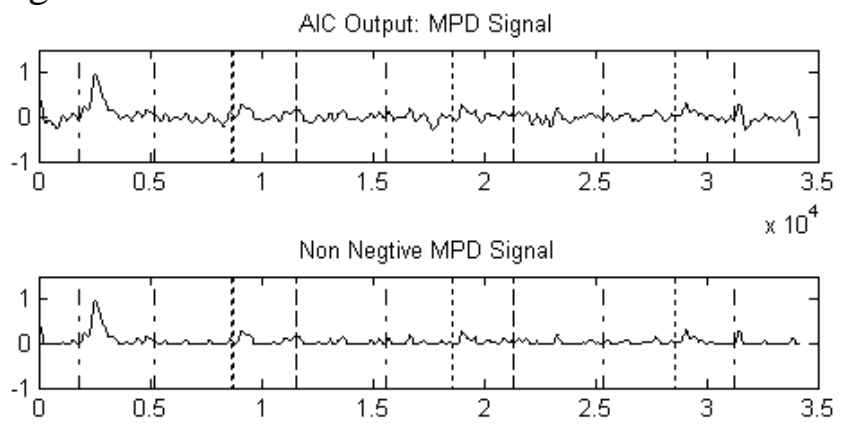

$\times 10^{4}$

PMPD: Sliding Window Median Analysis of Non-negative MPD

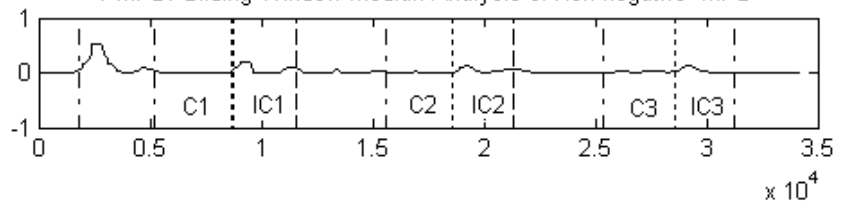

Figure 8. Example of the processing of the MPD signal (top panel) to obtain the PMPD signal (bottom panel). A non-negative constraint is first applied on MPD (shown in the middle panel), followed by median processing within a sliding window, which yields PMPD.

\subsection{ROC comparisons}

To assess the comparative discriminating capability of each of the features listed in Table 1, their individual ROC curves were plotted and an estimation of the area under each of them (AUROC) was obtained. It should be mentioned that the feature obtained as the segment average of the PMPD signal was only normalized through steps (2) and (3), because the illumination provided by the background of the TOBII screen during the relaxation period, before the $\mathrm{C} 1$ segment, is different from that provided during the rest of the protocol.

Table 2. AUROC results for all 10 features

\begin{tabular}{l|c}
\hline \multicolumn{1}{c|}{ Detection Signal } & AUROC \\
\hline PMPDmean & 0.9331 \\
\hline GSRmean. & 0.6780 \\
\hline GSRnum & 0.4683 \\
\hline GSRmeanAmp & 0.4337 \\
\hline GSRrisingTime & 0.5771 \\
\hline GSReng & 0.5023 \\
\hline BVPmeanAmp & 0.4189 \\
\hline BVPI2h & 0.4898 \\
\hline BVPIBImean & 0.3288 \\
\hline BVPstd & 0.4960 \\
\hline
\end{tabular}

The ROC curve for each of the 10 features was developed using their values from the 6 (segments) $\mathrm{x} 14$ (subjects) $=84$ segments analyzed in our study. Half of these segments correspond to "stressed" states induced by "IC" Stroop stimulation (ideal classifier output $=1$ ), and the other half are known to be associated with "relaxed" ("C" Stroop) intervals (ideal classifier output = -1). Each point of the ROC curves is determined by comparing the value of the single detection signal to a specific threshold and determining which portion of the "positive classifier outputs" match the ideal output (1) and which portion of the "negative classifier outputs" are in disagreement to the ideal output (-1). These "portions", expressed as fractional numbers, yield the coordinates of the ROC point (false positive rate, true positive rate) for the threshold value tested. The process was carried out using the ROC Matlab $®$ scripts provided by Dr. Gavin C. Cawley (University of East Anglia, Norwich, UK) in his web site http://theoval.sys.uea.ac.uk/matlab/ default.html. These scripts not only sweep the complete range of normalized threshold values, $[0$, 1], and draw the ROC, but additionally estimate a "convex hull" that fits the actual ROC points calculated. The convex hull is shown with dashed lines in the resulting plots. Additionally, the AUROC is calculated for each curve. The 
resulting AUROC values (Table 2) reveal that the PMPDmean feature has the highest discriminating power, followed distantly by the GSRmean feature. The ROC curves for these two features are presented in Figures 9 and 10.

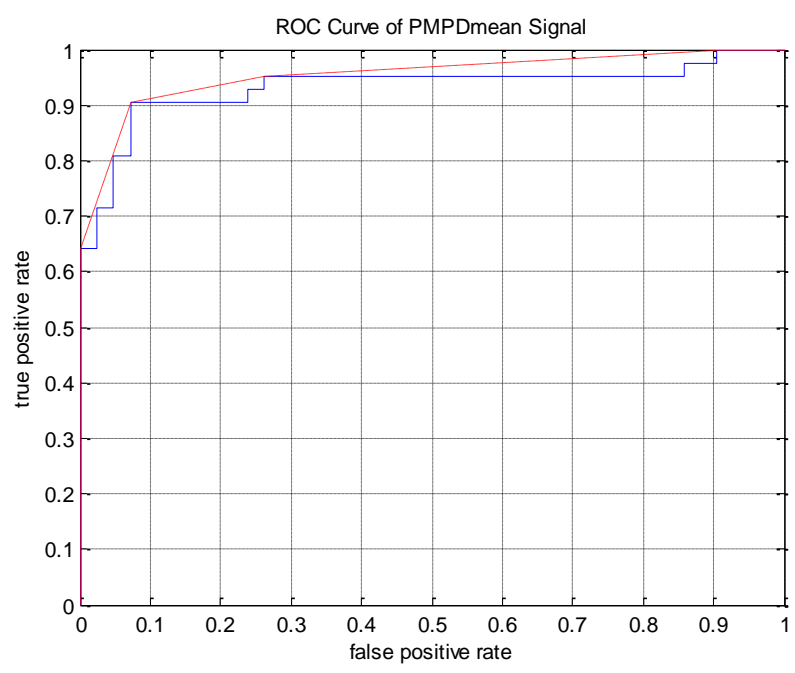

Figure 9. PMPDmean ROC curve. The closeness of the curve to the upper-left corner results in a high AUROC value (0.9331).

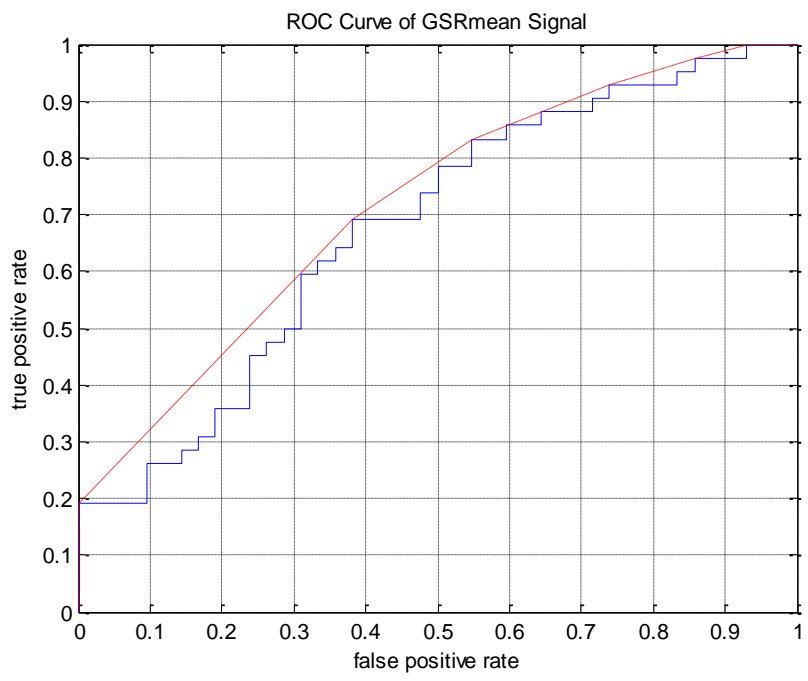

Figure 10. GSRmean ROC curve. The minimal convexity of the curve results in a moderate AUROC value (0.6780).

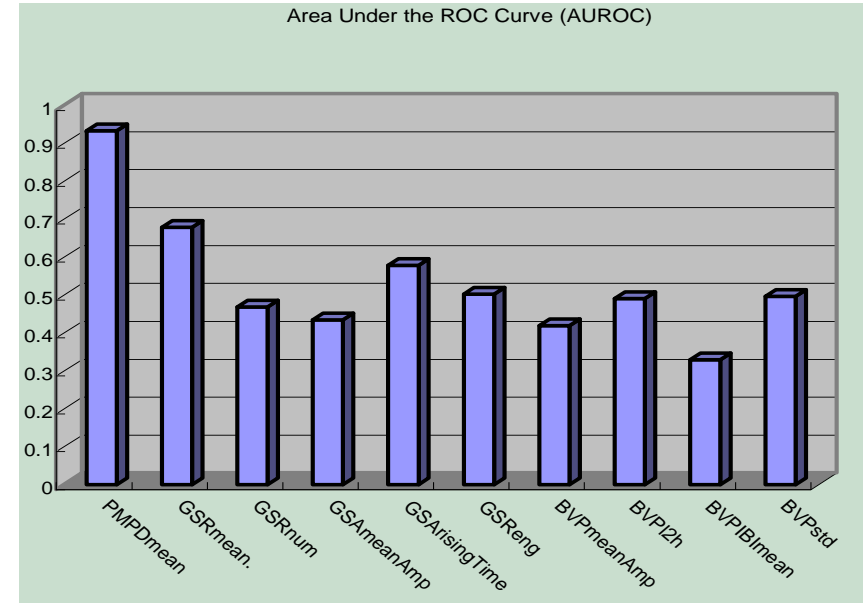

Figure 11. AUROC values for all 10 features. The PMPDmean feature resulted in an AUROC value that is clearly higher than the rest.

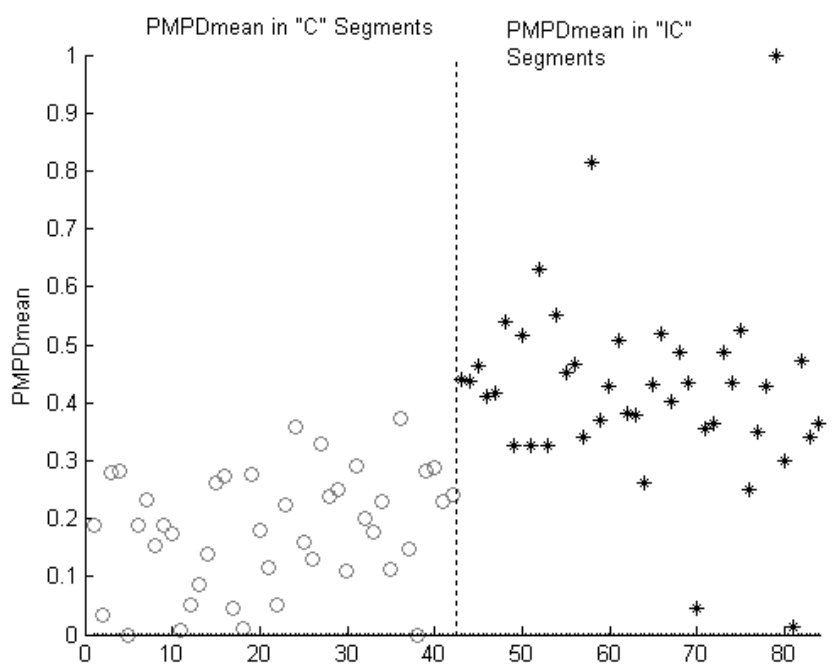

Figure 12. The distribution of PMPDmean feature values for both types of segments. It is apparent that the PMPDmean values for the two types of segments are scattered around different means.

\section{Discussion}

The ROC curve for GSRmean shows a convexity, which brings its area closer to 1 (AUROC_GSRmean $=0.6780)$. However, the ROC curve for PMPDmean, shown in Figure 9, exhibits an almost vertical increase from the coordinate origin, and reaches into high levels of true positive rate. Accordingly, the area under this 
curve is large: AUROC_PMPDmean $=0.9331$. Figure 11 shows a comparison of the area under the ROC curve (AUROC) for the 10 single-feature classifiers. This comparison emphasizes the higher discriminating power of the PMPDmean feature.

Furthermore, the separation between the values of the PMPDmean feature for congruent and incongruent segments can be visualized in the distribution plot shown in Figure 12. In this figure the values of the normalized PMDPmean feature for all the 42 Congruent (i.e., "relaxed") segments are plotted first, indicated with "O" markers, followed by the 42 PMDPmean values obtained from Incongruent (i.e., "stressed") segments, indicated with "*" markers. This plot confirms that, indeed, the clusters for both PMPDmean classes exhibit a definitive separation.

\section{Conclusion}

In this paper, we investigated the monitoring of a computer user's pupil diameter and its processing through an adaptive interference canceller (AIC) using the $\mathrm{H}^{\infty}$ time-Varying (HITV) adaptive algorithm. The goal was to extract only the Pupillary Affective Response (PAR) from the measured PD signal. Specifically, we use the output of the AIC, through further postprocessing, in order to detect the sympathetic activation associated with affective states such as stress.

A single-feature detector, driven by the PMPDmean feature, was evaluated in its potential to discriminate "Incongruent Stroop Segments", where the subject is expected to undergo stress, from "Congruent Stroop Segments", in which the subject is expected to remain relaxed. This evaluation was performed in terms of the Receiver Operating Characteristic curve of the singlefeature detector, which exhibited an area under the curve (AUROC) of 0.9331. In contrast, 9 other single-feature detectors driven by features extracted from the BVP and GSR signals exhibited much lower AUROC values, indicating that they have a lower potential to discriminate the Congruent and Incongruent Stroop segments.

The apparent performance advantage seen in the detector driven by a feature derived from the pupil diameter signal may be an important finding, since many contemporary affective sensing approaches only include the measurement of traditional signals, such as GSR and BVP, but few incorporate pupil diameter monitoring.

This study also addressed the challenge of retrieving only the pupil diameter changes related to affective responses, minimizing the impact of illumination changes (through the PLR) in the classification result. The encouraging results seen from the PMPDmean ROC were obtained in spite of intermittent illumination increases during the experiment. Therefore, these results confirmed the benefits of using the AIC implementation with the $\mathrm{H}^{\infty}$ time-varying adaptive algorithm to isolate the pupillary affective response.

\section{Acknowledgements:}

This work was sponsored by NSF grants CNS-0520811, HRD-0833093 and CNS-0426125. Ms. Ying Gao is the recipient of a Dissertation Year Fellowship from Florida International University.

\section{References}

1. Picard R.W. Affective Computing, MIT Press, Cambridge, Mass, 1997.

2. Hudlicka E. To feel or not to feel: the role of affect in human-computer interaction, International Journal of Human-Computer Studies, 2003, 59, 1-32.

3. Hugdahl, K. Psychophysiology: The MindBody Perspective, Harvard University Press, Cambridge, Mass, 1995.

4. Barreto, A.; Zhai, J.; Rishe, N.; Gao, Y. Significance of pupil diameter measurements for the assessment of affective state in computer users, Advances and Innovations in Systems, Computing Sciences and Software Engineering, K. Elleithy, ed., Springer, 2007, (ISBN: 978-1-4020-6263-6). Dordrecht, Netherlands, $59-64$.

5. Steinhauer S. R.; Siegle G. J.; Condray R.; Pless M. Sympathetic and parasympathetic innervation of pupillary dilation during sustained processing, International Journal of Psychophysiology,2004, 52, 77-86.

6. Bressloff P.C.; Wood C.V. Spontaneous 
oscillations in a nonlinear delayed-feedback shunting model of the pupillary light reflex, Physical Review E, 1998, 58, 3597-3605.

7. Partala, T.; Surakka, V. Pupil size variation as an indication of affective processing, Int. J. of Human-Computer Studies, 2003, 59, 185-198.

8. Widrow, B.; Stearns, S. D. Adaptive Signal Processing. Englewood Cliffs, NJ: PrenticeHall, 1985.

9. Stroop J. R.; 1935. Studies of interference in serial verbal reactions, Journal of Experimental Psychology, 1935, 18, 643-662.

10. Zhai J.; Barreto A. Stress detection in computer users through noninvasive monitoring of physiological signals. Biomedical Sciences Instrumentation, 2006, 42, 495-500.

11. Renaud P.; Blondin J.-P. The stress of Stroop performance: physiological and emotional responses to color-word interference, task pacing, and pacing speed, International Journal of Psychophysiology, 1997, 27, 8797.

12. Stern, R.M.; Ray, W. J.; Quigley, K.S., Psychophysiological Recording. Oxford University Press, 2001.
13. Todoroki A.; Hana N. Luminance change method for cure monitoring of GFRP, Key Engineering Materials, 2006, 321-323, 13161321.

14. Puthusserypady, $\mathrm{S} . \mathrm{H}^{\infty}$ adaptive filters for eye blink artifact minimization from electroencephalogram. IEEE Signal Processing Letters, 2005, 12, 816-819.

15. Hassibj, B.; Kailath T. $\mathrm{H}^{\infty}$ adaptive filtering, Proc. Int. Conf. Acoustics, Speech, Signal Processing, 1995, 945-952.

16. Barreto, A.; Zhai, J.; Adjouadi M. Nonintrusive physiological monitoring for automated stress detection in humancomputer interaction, Lecture Notes in Computer Science, Springer-Verlag, 2007, 4796, 29-38,.

17. Beatty J.; Lucero-Wagoner B. The pupillary system, Handbook of Psychophysiology, Cacioppo, Tassinary \& Berntson, Cambridge University Press, 2000. 